\title{
Leishmaniose Tegumentar Americana: Uma Doença Polimorfa
}

\author{
Charles Name De Dominicis', Flávia Regina Ferreira², Fátima Maria de Oliveira Rabay ${ }^{3}$, Samuel Henrique Mandelbaum ${ }^{4}$ \\ 'Especialista em Clínica Médica pela Sociedade Brasileira de Clínica Médica (SBCM) - (AMB) - Médico - Especializando do $3^{\circ}$ \\ ano de Dermatologia do Serviço de Dermatologia do Hospital Universitário de Taubaté - SP, Brasil \\ ${ }^{2}$ Mestre e Doutora em Ciências pela Universidade Federal de São Paulo/Escola Paulista de Medicina - UNIFESP/EPM - Professora \\ Assistente da Disciplina de Dermatologia do Departamento de Medicina da Universidade de Taubaté/Médica do Serviço de \\ Dermatologia do Hospital Universitário de Taubaté-Taubaté-SP. Brasil \\ ${ }^{3}$ Fátima Maria de Oliveira Rabay - Especialista em Dermatologia pela Sociedade Brasileira de Dermatologia - Professora Assistente \\ da Disciplina de Dermatologia do Departamento de Medicina da Universidade de Taubaté/Médica do Serviço de Dermatologia do \\ Hospital Universitário de Taubaté-Taubaté-SP. Brasil \\ ${ }^{4}$ Samuel Henrique Mandelbaum - Especialista em Dermatologia pela Sociedade Brasileira de Dermatologia - Professor \\ da Disciplina de Dermatologia do Departamento de Medicina da Universidade de Taubaté/Médico e Chefe do Serviço de \\ Dermatologia do Hospital Universitário de Taubaté-Taubaté-SP. Brasil
}

RESUMO - Pela importância mundial que o tema repercute apresentamos um caso de leishmaniose tegumentar Americana num paciente de 65 anos com envolvimento restrito a pele, apesar da sua longa evolução (> 3 anos). Trata-se de um caso de difícil diagnóstico pela apresentação clínica atípica (úlcera de grandes dimensões) e estudo histopatológico inconclusivo nas biópsias iniciais. A terceira biópsia com observação cuidada e com auxilio de estudo imunohistoquímico permitiram o diagnóstico correcto e cura após 30 administrações ev de antimoniato de $\mathrm{N}$-metilglucamina.

PALAVRAS-CHAVE - Leishmania braziliensis; Leishmaniose Cutânea.

\section{American Cutaneous Leishmaniasis: A Polymorphic Disease}

ABSTRACT - Due to the worldwide importance of this theme, we present a case of American cutaneous leishmaniasis in a 65-year-old patient with restricted skin involvement, despite its long evolution (> 3 years). It was a difficult case to diagnose due to the atypical clinical presentation (large ulcer) and inconclusive histopathological study in the initial biopsies. The third biopsy with careful observation and the contribution of an immunohistochemical study allowed a correct diagnosis and cure after 30 administrations of $\mathrm{N}$-methylglucamine antimoniate ev.

KEYWORDS - Leishmaniasis, Cutaneous; Leishmania braziliensis.

\section{INTRODUÇ̃̃O}

A Leishmania é um parasita intracelular obrigatório, um protozoário que possui uma forma flagelada alongada e móvel, encontrada no flebótomo ou em meios de cultura artificiais, e uma forma aflagelada, que aparece como pequenos corpos esféricos ou amastigotas, conhecidos como corpos de Leishman-Donovan observados no interior de macrófagos infectados. Tradicionalmente, existem três formas principais de leishmaniose: cutânea, mucocutânea e visceral.

A leishmaniose tegumentar Americana (LTA) caracteriza-se principalmente pelo comprometimento do tecido cutâneo e, secundariamente, do tecido mucoso naso-buco-faríngeo, dependendo a forma de apresentação da espécie de Leishmania e de fatores imunogenéticos do hospedeiro. Esta
Correspondência: Charles Name De Dominicis Hospital Universitário de Taubaté

Avenida Granadeiro Guimarães, 270 - Centro - Taubaté - SP

CEP: 12020-130

E-mail: charlesdominicis@yahoo.com.br

DOI: https://dx.doi.org/10.29021/spdv.76.2.840
Recebido/Received

31 Outubro/October 2017

Aceite/Accepted

02 Janeiro/January 2018 


\section{Caso Clínico}

doença infecciosa, crônica não contagiosa pode ser causada por diferentes espécies de Leishmania transmitidas de animais para o homem por fêmeas de flebotomíneos infectadas. As espécies mais comuns no Brasil são a Leishmania (Viannia) brasiliensis, a Leishmania (Viannia) guyanensis e a Leishmania (Mexicana) amazonensis. Ainda que em 95\% dos casos se manifeste por uma úlcera característica com bordo bem delimitado, infiltrado, saliente (em moldura de quadro) e fundo granuloso, avermelhado, exsudativo, praticamente indolor, é considerada uma doença polimorfa, frente à variedade de outras apresentações: impetigoide, ectimatoide, vegetante, verrucosa, infiltrativa, nodular, tuberosa, lupoide e esporotricoide. ${ }^{1,2}$

A apresentação e evolução atípicas deste caso e a dificuldade diagnóstica encontrada nos motivaram neste relato.

\section{CASO CLÍNICO}

Paciente masculino, 67 anos, caucasiano, que procurou - Serviço de Dermatologia por úlcera extensa de bordos mal delimitados, fundo granuloso e secreção amarelada, ocupando grande parte do punho e face lateral da mão direita, com evolução de 3 anos e meio (Fig. 1). A lesão inicial era uma úlcera, indolor que surgiu cerca de 30 dias após trauma local com capim (dados reportados pelo paciente). Após observação noutro Serviço de Dermatologia e exames

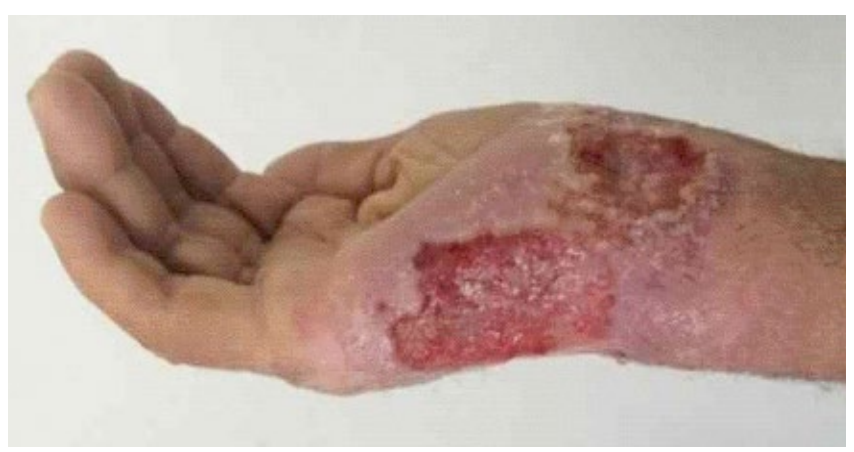

Figura 1 - Punho e face lateral da mão direita - úlcera extensa de bordos mal delimitados, fundo granuloso e com secreção amarelada. histopatológicos que favoreciam esporotricose encontrava-se em tratamento com Itraconazol $200 \mathrm{mg} /$ dia há 11 meses, sem melhora. Referia apenas hipertensão arterial e tabagismo e negava outras doenças ou história de imunossupressão.

Realizamos nova biópsia (leito e bordo da úlcera) mas o exame histopatológico foi inconclusivo, revelando apenas infecção secundária e afastando a hipótese de carcinoma espinocelular. As culturas para fungos e micobactérias foram negativas. Empiricamente foi iniciado teste terapêutico, com iodeto de potássio na dose inicial de $0,5 \mathrm{~g} / \mathrm{dia}$ a qual foi aumentada gradualmente até $4 \mathrm{~g} /$ dia (a cada 2-4 semanas), suspenso após 3 meses por ausência de resposta.

Optou-se, então, por abstenção terapêutica durante 3 meses antes de reiniciar a investigação. Foram realizadas novas biópsias com bisturi circular de 3 diferentes pontos da lesão. $\bigcirc$ estudo histopatológico evidenciou formas amastigotas dentro dos macrófagos e o estudo imunohistoquímico revelou marcação para corpos de Leishman (Fig. 2). Não foi efectuada cultura ou PCR, pelo que a espécie de Leishmania envolvida não foi caracterizada. Do exame geral não eram evidentes adenomegalias, lesões das mucosas e os exames complementares como: hemograma, glicemia em jejum, lipidograma, anti-VIH, função tireoidiana, hepática e renal não revelaram alterações.

Foi iniciada terapia endovenosa com antimoniato de $\mathrm{N}$ -metilglucamina, em ampolas de $5 \mathrm{~mL}$ correspondendo a $405 \mathrm{mg}$ de antimónio pentavalente administradas diariamente sem diluição, com agulha fina (calibre $25 \times 8$ ) e de forma lenta (aproximadamente 5 minutos). Durante todo o tratamento o paciente foi monitorado através de eletrocardiograma e foi realizada vigilância laboratorial das enzimas hepáticas e da função renal. Após 30 doses foi observada a cura clínica da lesão. Na Fig. 3, evolução após 2 meses do término da terapia. $\bigcirc$ paciente está há 09 meses em cura clínica e sem sinais de recidiva.

\section{DISCUSSÃO E CONCLUSÃO}

A LTA é um problema de saúde pública, já que se estima incidência anual de aproximadamente dois milhões de

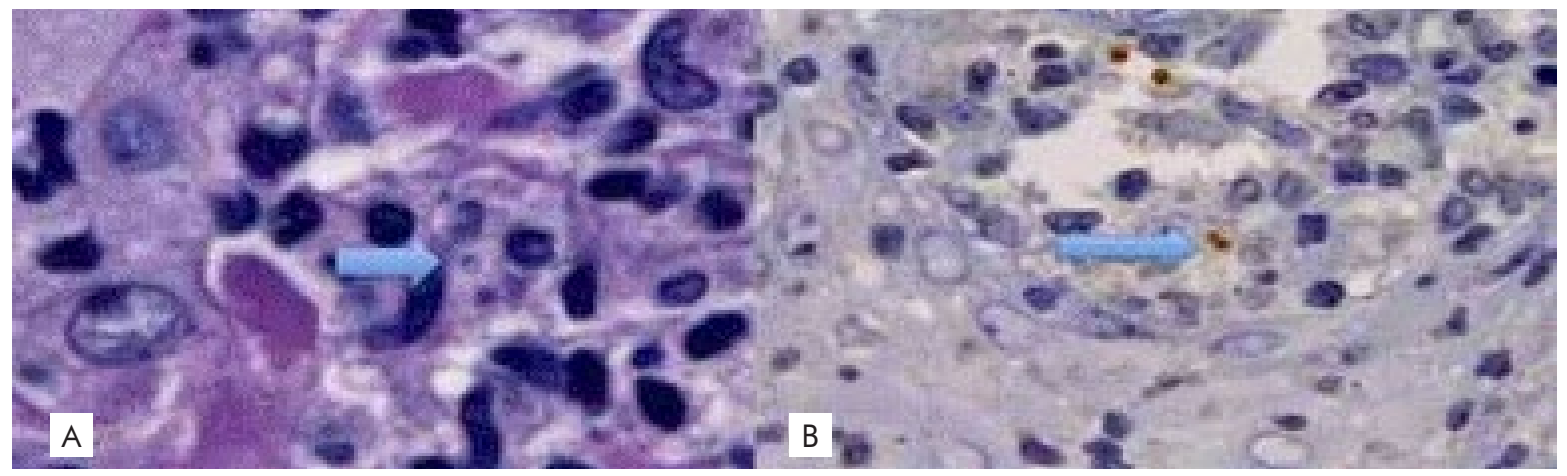

Figura 2 - (A) Na seta formas amastigotas de Leishmania no interior do citoplasma de macrófagos (H\&E, 1000X)/ (B) - Imunohistoquímica - na seta marcação positiva para os corpos de Leishman (1000X). 


\section{Caso Clínico}

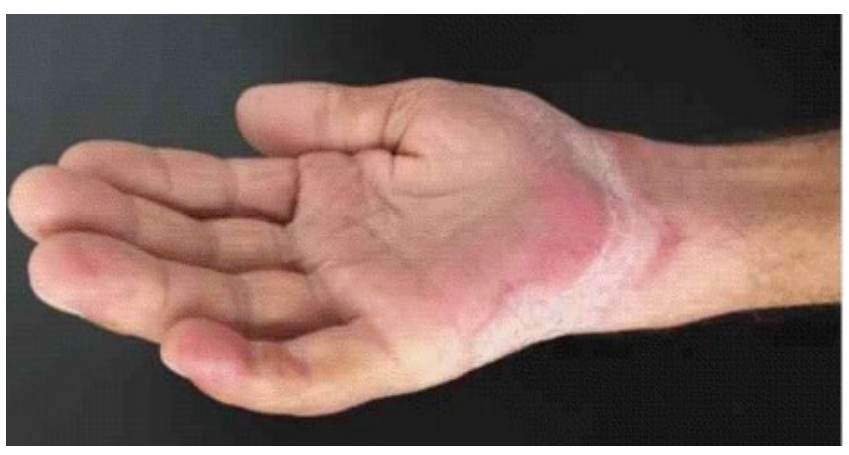

Figura 3 - Cura clínica após 30 doses do Antimoniato de N-metilglucamina.

casos novos e 12 milhões de pessoas infectadas no mundo. Dados da Organização Mundial de Saúde (OMS), apontam que 350 milhões de indivíduos estão expostos ao risco de infecção pelo gênero Leishmania. ${ }^{3}$

A LTA é tida como uma doença polimorfa como demonstra o presente caso que não apresentou a úlcera clássica com borda em moldura de quadro. Classicamente afeta apenas a pele mas pode ocorrer comprometimento mucoso, simultaneamente com as lesões de pele ou, o que é mais frequente, meses ou anos após a cicatrização das lesões cutâneas. Inicia-se por eritema e discreta infiltração da mucosa, seguido de granulação e ulceração, chegando por último, à perfuração do tecido cartilaginoso do septo nasal ou do palato. ${ }^{4}$ Mesmo com um tempo de evolução longo (> 3 anos) neste paciente não foi detectado acometimento mucoso buco-naso-faríngeo, cujo risco aumenta em lesões de longa duração. ${ }^{5}$

O diagnóstico da LTA pode ser obtido por provas diretas e indiretas. Diretas: o isolamento do parasito em meio de cultura apropriado (NNN, Difco-bifásico B45, LIT, entre outros), dependente não só do tempo da lesão, mas também do tipo da lesão. O exame parasitológico direto, obtido por escarificação ou aspirado da borda interna da úlcera e a colocação do esfregaço sobre a superfície de uma lâmina, fixado com metanol a $70 \%$ e, em seguida, corado com solução de Giemsa, Leishman ou Wright. O exame histopatológico é fidedigno quando mostra a presença de formas amastigotas no interior do citoplasma de macrófagos na derme, mas pode ser confirmado por estudo imunohistoquímico (como no presente caso) ou por PCR. Nos exames indiretos, temos: a reação intradérmica de Montenegro, reação sorológica de imunofluorescência indireta (RIFI), ensaio imunoenzimático (ELISA). ${ }^{1,6,7}$

Neste caso, contamos, com os aspectos: epidemiológico, clínico, histopatológico e imunohistoquímico para elucidar o diagnóstico e orientar o tratamento correto deste paciente, com o antimoniato de $\mathrm{N}$-metilglucamina, droga de primeira escolha para a terapia da LTA.4,9 Após, 30 doses, observamos a cura clínica da lesão. A opção pelas 30 doses e não 20, conforme preconizado para formas cutâneas, se deu por um excesso de zelo dos autores preocupados com longo tempo de evolução e possível disseminação extra-cutânea do parasita. ${ }^{10,11-16}$

O caráter muitas vezes desfigurante, destrutivo e limitante das lesões da LTA, impactando na qualidade de vida dos indivíduos nos motivou a descrever este relato, ressaltando a apresentação exuberante e atípica deste paciente, a dificuldade diagnóstica e a importância da persistência na busca pelo diagnóstico que nos permitiu sucesso terapêutico.

Agradecimentos: Agradecemos às patologistas Fernanda Rocha Gonçalves e Márcia Lanzoni de Alvarenga Lira o auxílio no diagnóstico deste caso.

Conflitos de interesse: Os autores declaram não possuir conflitos de interesse.

Suporte financeiro: $O$ presente trabalho não foi suportado por nenhum subsídio ou bolsa.

Confidencialidade dos dados: Os autores declaram ter seguido os protocolos do seu centro de trabalho acerca da publicação dos dados de doentes.

Protecção de pessoas e animais: Os autores declaram que os procedimentos seguidos estavam de acordo com os regulamentos estabelecidos pelos responsáveis da Comissão de lnvestigação Clínica e Ética e de acordo com a Declaração de Helsínquia da Associação Médica Mundial.

Conflicts of interest: The authors have no conflicts of interest to declare.

Financing Support: This work has not received any contribution, grant or scholarship.

Confidentiality of data: The authors declare that they have followed the protocols of their work center on the publication of data from patients.

Protection of human and animal subjects: The authors declare that the procedures followed were in accordance with the regulations of the relevant clinical research ethics committee and with those of the Code of Ethics of the World Medical Association (Declaration of Helsinki).

\section{REFERÊNCIAS}

1. Belda Junior W, Di Chiacchio Di N, Criado PR. Tratado de Dermatologia. $2^{a}$ ed. São Paulo: Atheneu; 2014.

2. Ejaz A, Raza N, Iftikhar N. Recurrent cutaneous leishmaniasis presenting as sporotrichoid abscesses: $\mathrm{A}$ rare presentation near Afghanistan border. Dermatol Online J. 2007; 13:15.

3. David Azulay R, Rubem Azulay D, Azulay Abulafia L. Azulay Dermatologia. $6^{a}$ ed Rio de Janeiro: Guanabara Koogan; 2015.

4. Ourives-Neves L, Chrusciak-Talhari A, Gadelha EPN, da Silva Júnior RM, Guerra JA, Ferreira LC, et al. A randomized clinical trial comparing meglumine antimoniate, pentamidina and amphotericin B for the treatment of cutaneous leishmaniasis by Leishmania guyanensis. An Bras Dermatol. 2011; 86:1092-101.

5. Brasil, Ministério da Saúde, Secretaria de Vigilância em Saúde, Departamento de Vigilância Epidemiológica. 


\section{Caso Clínico}

Manual de Vigilância da Leishmaniose Tegumentar Americana. $2^{a}$ ed. - Brasília: Editora do Ministério da Saúde; 2013.

6. Couto DV, Hans-Filho G, Medeiros MZ, Vicari CFS, Barbosa AB, Takita LC. Leishmaniose tegumentar americana - um caso de desafio terapêutico. An Bras Dermatol. 2014; 89:978-80.

7. Araujo LM, Maia DC, Agonio B, Cerdan LM, Galvão RS, Francesconi do Valle VA, et al. Leishmaniose tegumentar americana - apresentação atípica diagnosticada com técnica de biologia molecular. Rev Soc Port Dermatol Venereol. 2015; 73:479-83.

8. Lima MV, Oliveira RZ, Lima AP, Cerino DA, Silveira TG. American cutaneous leishmaniasis with fatal outcome during pentavalent antimoniate treatment. An Bras Dermatol. 2007; 82:269-71.

9. Miltropoulos P, Konidas P, Durkin-Konidas M. New World cutaneous leishmaniasis: update review of current and future diagnosis and treatment. J Am Acad Dermatol. 2010; 63:309-22.

10. Machado-Pinto J, Pinto i, Costa CA, Genaro O, Marques MJ, Modabber F, et al. Immunochemotherapy for cutaneous leishmaniasis: a controlled trial using killed Leishmania amazonensis vaccine plus antimonial. Int J
Dermatol. 2002; 41:73-8.

11. Marsden PD, Sampaio RN, Carvalho EM, Veiga JP, Costa JL, Llanos-Cuentas EA. High continuous Antimony therapy in two patients with unresponsive mucosal leishmaniasis. Am J Trop Med Hyg. 1985; 34:710-3.

12. Mattos MS, Friedman RK, Silva Filho IL, Oliveira-Neto MP. Pancreatite: um dos efeitos adversos da terapia antimonial na leishmaniose. An Bras Dermatol. 1991; 75:45-50.

13. Mayrink W, Michlick MS, Melo MN, Williams P, Nascimento E, Araújo P, et al. Tratamento da leishmaniose tegumentar Americana utilizando vacina. An Bras Dermatol. 1991; 66:55-9.

14. González U. Fluconazol for cutaneous leishmaniosis: looking for a better treatment. Arch Dermatol. 2002; 138:1604-6.

15. Lima MV, Oliveira RZ, Lima AP, Cerino DA, Silveira TG. Leishmaniose cutânea com desfecho fatal durante o tratamento com antimonial pentavalente. An Bras Dermatol. 2007; 82:269-71.

16. Sadeghian G, Nilforoushzadeh MA. Effect of combination therapy with systemic glucantime and pentoxifylline in the treatment of cutaneous leishmaniasis. Int J Dermatol. 2006; 45:819-21. 